\title{
Baixas Doses de Bupivacaína Hipobárica para Raquianestesia Unilateral *
}

\section{Low Hypobaric Bupivacaine Doses for Unilateral Spinal Anesthesia}

\author{
Luiz Eduardo Imbelloni, TSA ${ }^{1}$; Lúcia Beato, TSA ${ }^{1}$; M A Gouveia, TSA ${ }^{2}$
}

\author{
RESUMO \\ Imbelloni LE, Beato L, Gouveia MA - Baixas Doses de Bupiva- \\ caína Hipobárica para Raquianestesia Unilateral
}

JUSTIFICATIVA E OBJETIVOS: Para evitar alterações hemodinâmicas, obter recuperação mais rápida e limitar a dispersão cefálica da raquianestesia apenas no membro operado foi realizado este estudo com bupivacaína a 0,15\%, com objetivo de se obter raquianestesia unilateral.

MÉTODO: Raquianestesia com $3,3 \mathrm{ml}$ de bupivacaína hipobárica a 0,15\% (5 mg) foi realizada através de agulha $27 \mathrm{G}$ Quincke em 20 pacientes estado físico ASA I e II submetidos a cirurgias ortopédicas. A punção subaracnóidea foi realizada por via lateral com o paciente em decúbito lateral, com o membro a ser operado voltado para cima, e 3,3 $\mathrm{ml}$ de bupivacaína hipobárica foram injetados na velocidade de $1 \mathrm{ml}$ a cada 15 segundos. Bloqueios sensitivo e motor (picada de agulha e escala de 0 a 3) foram comparados entre os lados a ser operado e o contralateral.

RESULTADOS: Os bloqueios motor e sensitivo entre o lado operado e o contralateral foram significativamente diferentes em todos os tempos avaliados. Raquianestesia unilateral foi obtida em $75 \%$ dos pacientes. Estabilidade hemodinâmica foi observada em todos os pacientes. Nenhum paciente desenvolveu cefaléia pós-raquianestesia.

CONCLUSÕES: A bupivacaína hipobárica a 0,15\% na dose de $5 \mathrm{mg}$ proporciona um predominante bloqueio unilateral. Vinte minutos são suficientes para a sua instalação. A principal vantagem da raquianestesia unilateral é a estabilidade hemodinâmica.

Unitermos: ANESTÉSICOS, Local: bupivacaína; TÉCNICAS ANESTÉSICAS, Regional: subaracnóidea

\author{
SUMMARY \\ Imbelloni LE, Beato L, Gouveia MA - Low Hypobaric Bupivacai- \\ ne Doses for Unilateral Spinal Anesthesia
}

BACKGROUND AND OBJECTIVES: The possibility to achieve unilateral spinal anesthesia with $0.15 \%$ bupivacaine was studied with the purpose of minimizing hemodynamic changes, limiting the cephalad dispersion of the anesthetic and promoting a faster recovery.

METHODS: Twenty ASA I - II patients undergoing orthopedic surgeries were given spinal $0.15 \%$ hypobaric bupivacaine through a 27G Quincke needle. Dural puncture was performed with patients in the lateral position, with the limb to be operated upwards, and $3.3 \mathrm{ml}(5 \mathrm{mg})$ hypobaric bupivacaine were injected at the rate of $1 \mathrm{ml} .15 \mathrm{~s}^{-1}$. Sensory and motor block (pinprick and 0 to 3 scale) were compared between operated and contralateral sides.

RESULTS: Motor and sensory block in operated and contralateral sides were significantly different in all evaluated times. Unilateral spinal anesthesia was achieved in $75 \%$ of patients. All patients remained hemodynamically stable, and no one developed post-dural puncture headache.

CONCLUSIONS: Hypobaric bupivacaine $(5 \mathrm{mg})$ is able to provide a predominant unilateral block with the patient being kept twenty minutes in the lateral position. Major unilateral spinal anesthesia advantage is hemodynamic stability.

Key Words: ANESTHETICS, Local, bupivacaine; ANESTHETIC TECHNIQUES, Regional: spinal block

\section{INTRODUÇÃO}

A raquianestesia é um dos mais antigos métodos de alívio da dor em pacientes cirúrgicos. Freqüentemente são realizados procedimentos cirúrgicos envolvendo apenas um membro inferior, particularmente em pacientes ambula-

\footnotetext{
* Recebido da (Received from) Casa de Saúde Santa Maria, Clínica São Bernardo e Hospital do IASERJ, Rio de Janeiro, RJ

1. Anestesiologista da Casa de Saúde Santa Maria e Clínica São Bernardo. Rio de Janeiro

2. Chefe do Serviço de Anestesiologia do Hospital Central do IASERJ, Rio de Janeiro
}

Apresentado (Submitted) em 23 de setembro de 2002

Aceito (Accepted) para publicação em 11 de janeiro de 2003

Endereço para correspondência (Correspondence to)

Dr. Luiz Eduardo Imbelloni

Av. Epitácio Pessoa, 2356/203 Lagoa

22471-000 Rio de Janeiro, RJ

E-mail: imbelloni@openlink.com.br

(C) Sociedade Brasileira de Anestesiologia, 2003

Revista Brasileira de Anestesiologia

Vol. 53, № 5, Setembro - Outubro, 2003 toriais e cirurgias ortopédicas. Nestas situações, a raquianestesia unilateral apresenta algumas vantagens em relação à raquianestesia convencional que são redução da hipotensão arterial ${ }^{1}$, recuperação mais rápida do bloqueio ${ }^{1}$ e aumento da satisfação do paciente ${ }^{2}$. Existem diversas razões para se controlar o nível do bloqueio sensitivo máximo. Cirurgias de membros inferiores necessitam de baixos níveis de bloqueio sensitivo, resultando em maior estabilidade cardiocirculatória ${ }^{3}$. Com pequenas doses de anestésicos locais podem ser evitados efeitos indesejáveis, como bloqueio motor prolongado, instabilidade hemodinâmica e retenção urinária ${ }^{4,5}$. O decúbito lateral, baixas doses de anestésico local, orientação da agulha ponta de lápis e injeção lenta de anestésico têm sido sugeridos para facilitar a obtenção de raquianestesia unilateral ${ }^{6,7}$. Em estudo prévio ${ }^{2}$ foi demonstrado que a solução hipobárica de bupivacaína a $0,15 \%$, agulha ponta cortante e retirada de um volume de 3 a $5 \mathrm{ml}$ de líquido cefalorraquidiano (LCR) produziram raquianestesia unilateral em $71 \%$ dos pacientes. 
O objetivo desta investigação foi avaliar a tentativa de distribuição assimétrica do bloqueio entre o membro a ser operado e o contralateral e a influência na incidência de hipotensão arterial durante a raquianestesia para procedimentos ortopédicos de membros inferiores com baixas doses de bupivacaína $0,15 \%$ hipobárica sem retirada de LCR.

\section{MÉTODO}

Após aprovação da Diretoria de Publicação e Divulgação das Clínicas e consentimento informado, participaram deste estudo 20 pacientes estado físico ASA I e II, com idades entre 21 e 79 anos, submetidos à raquianestesia para cirurgias ortopédicas eletivas e de urgências envolvendo apenas um dos membros. Critérios de exclusão foram hipovolemia, distúrbios de coagulação, infecção e recusa do método proposto. A monitorização incluiu avaliação da pressão arterial por método não invasivo, freqüência cardíaca e oximetria de pulso. Não foi administrada nenhuma medicação pré-anestésica. Após a chegada à sala de operação foi instalada venóclise com solução de Ringer com lactato e injetados 25 a $50 \mu \mathrm{g}$ de fentanil.

O paciente foi colocado em decúbito lateral com o membro a ser operado voltado para cima. A coluna vertebral foi posicionada horizontalmente antes da punção subaracnóidea e a mesa nivelada na posição horizontal (zero) durante o procedimento. A punção subaracnóidea foi realizada no espaço $\mathrm{L}_{3}-\mathrm{L}_{4}$ com agulha $27 \mathrm{G}$ tipo Quincke pela via paramediana e injetados $5 \mathrm{mg}$ de bupivacaína hipobárica a 0,15\% (preparada a partir de 7,5 mg da bupivacaína a $0,5 \%$ isobárica acrescida de $3,5 \mathrm{ml}$ de água bidestilada) (Tabela I) na velocidade de $1 \mathrm{ml}$ a cada 15 segundos com a ponta orientada para o membro superior. O paciente foi mantido nesta posição por 20 minutos e em seguida colocado em decúbito dorsal horizontal.

Tabela I - Avaliação da Solução Injetada no LCR *

\begin{tabular}{lcc}
\hline & $20^{\circ} \mathrm{C}$ & $37^{\circ} \mathrm{C}$ \\
\hline Densidade $\left(\mathrm{g} \cdot \mathrm{ml}^{-1}\right)$ & 1,001 & 0,996 \\
Osmolaridade $\left(\mathrm{mOsm} \cdot \mathrm{kg}^{-1}\right)$ & 149 & 149 \\
$\mathrm{pH}$ & 5,28 & 5,10 \\
Teor de bupivacaína $\left(\mathrm{mg} \cdot \mathrm{ml}^{-1}\right)$ & 7,585 & 7,585 \\
Água para injetáveis q.s.p. $(\mathrm{ml})$ & - & 3,5 \\
\hline
\end{tabular}

* Cristália Produtos Químicos e Farmacêuticos Ltda

O nível sensitivo foi avaliado pela perda da sensação de frio (gelo) e picada de agulha 27G, enquanto que o bloqueio motor foi avaliado pela escala modificada de Bromage $(0=\mathrm{sem}$ bloqueio motor a 3 = bloqueio motor completo). A avaliação dos bloqueios sensitivo e motor foi realizada em ambos os membros aos 20, 40 e 60 minutos e ao final da cirurgia. Os parâmetros hemodinâmicos foram avaliados a cada três minutos durante os primeiros 15 minutos após a punção subaracnóidea e posteriormente a cada cinco minutos até o final da cirurgia.

A hipotensão arterial foi definida como a diminuição de $30 \%$ dos valores basais, enquanto que bradicardia foi definida como FC menor que 50 bpm. A hipotensão arterial seria tratada com $1 \mathrm{mg}$ de etilenofenilefrina e bradicardia com $0,5 \mathrm{mg}$ de atropina, por via venosa. Durante o procedimento, um garrote pneumático foi inflado $100 \mathrm{mmHg}$ acima da pressão arterial sistólica. Um fluxo de 2 L. min $^{-1}$ de oxigênio foi administrado através de cateter nasal ou máscara de Hudson. Midazolam (1,5 a $3 \mathrm{mg})$ foi administrado após a segunda avaliação (40 minutos).

Os pacientes foram acompanhados até o terceiro dia de pós-operatório para se obter informação sobre sua satisfação com a técnica e para pesquisa de cefaléia pós-punção ou sintomas neurológicos transitórios (SNT), e até o $30^{\circ}$ dia com relação às complicações neurológicas definitivas.

As variáveis quantitativas foram analisadas em termos de estatística descritiva, sendo as comparações feitas com o teste não paramétrico de Kruskal-Wallis e as comparações dos bloqueios nos dois membros pelo teste não paramétrico do sinal. O nível de significância adotado foi de $5 \%$.

\section{RESULTADOS}

Os dados demográficos dos pacientes estão apresentados na tabela II.

Tabela II - Dados Demográficos, Duração da Cirurgia e do Bloqueio Sensitivo

\begin{tabular}{lc}
\hline Idade (anos) * & $41,6 \pm 17$ \\
Peso $(\mathrm{kg}){ }^{*}$ & $65,6 \pm 16,1$ \\
Altura $(\mathrm{cm}){ }^{*}$ & $163,3 \pm 8,2$ \\
Sexo & 12 \\
$\quad$ Feminino & 8 \\
$\quad$ Masculino & $1,2 \pm 0,2$ \\
Duração da cirurgia (horas) * & $2,32 \pm 0,16$ \\
\hline Duração do bloqueio (horas) * &
\end{tabular}

Bloqueio unilateral completo foi observado em $75 \%$ dos pacientes. Aregressão completa do bloqueio ocorreu, em média, com 2,32 h. Não houve diferença significante na regressão completa do bloqueio quanto ao sexo (valor $p=0,21$ ), nem na duração da cirurgia (valor $p=0,88$ ).

A dispersão do bloqueio sensitivo do lado do membro operado variou de $T_{7} a L_{1}$ sendo a moda igual a $T_{12}$. No membro contralateral aos 20 minutos, quatro pacientes apresentaram bloqueio sensitivo que variou de $T_{10}$ a $L_{1}$. Aos 40 minutos, mais um paciente relatou bloqueio sensitivo em $\mathrm{T}_{12}$. Portanto, o bloqueio sensitivo unilateral ocorreu em 15 pacientes ( $75 \%$ ) e em ambos os membros em 5 pacientes (25\%). O bloqueio no membro operado foi significativamente maior. 


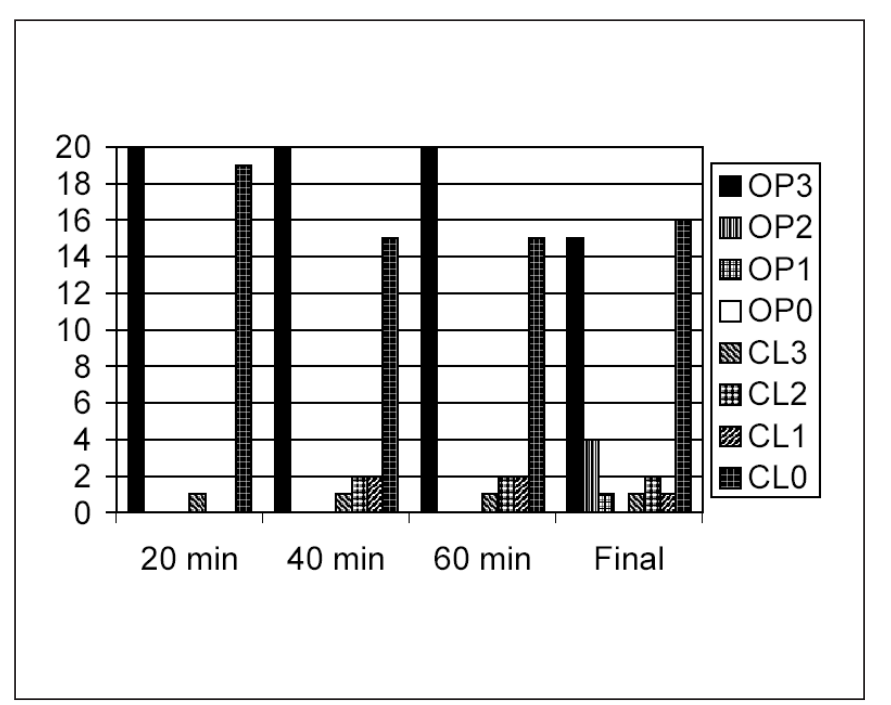

Figura 1 - Bloqueio Motor no Lado Operado e Contralateral nos Diferentes Tempos

* Bupivacaína a $0,15 \%$ gentilmente produzida e avaliada pela Cristália Produtos Farmacêuticos Ltda

Em todos os pacientes ocorreu bloqueio motor completo no membro operado. Um paciente apresentou bloqueio motor completo no membro contralateral. Ocorreu bloqueio motor grau 2 em 2 pacientes e grau 1 em 2 pacientes (Figura 1). $O$ bloqueio motor unilateral ocorreu em 15 pacientes $(75 \%)$. $O$ bloqueio motor no membro operado foi maior que no membro contralateral em cada avaliação.

Em todos os pacientes, a raquianestesia foi satisfatória para o procedimento e nenhum paciente necessitou de complementação com anestesia geral. Um paciente referiu dor ao torniquete aos 85 minutos de cirurgia. Não ocorreram alterações da pressão arterial e da freqüência cardíaca em nenhum paciente. Não se observou retenção urinária nos pacientes estudados e nenhum paciente desenvolveu cefaléia pós-raquianestesia. Não houve relato de dor nas costas, nádegas ou pernas que pudessem ser classificados como sintomas neurológicos transitórios, nem ocorreram complicações neurológicas tardias.

Não ocorreu falha da anestesia. Todos os pacientes ficaram satisfeitos com a técnica. Os 15 pacientes que apresentaram raquianestesia unilateral relataram sensação agradável em permanecer sentindo o membro contralateral.

\section{DISCUSSÃO}

Baixas doses de bupivacaína hipobárica $(5 \mathrm{mg})$ na velocidade de $1 \mathrm{ml}$ a cada 15 segundos através de agulha 27G Quincke produziram bloqueio unilateral completo em $75 \%$ dos pacientes. Araquianestesia unilateral demonstrou ser segura e efetiva em cirurgias ortopédicas de um membro, reduzindo o impacto hemodinâmico dos bloqueios realizados no neuro-eixo ${ }^{1,2,8}$ e aumentando a satisfação dos pacientes com a técnica.

Adensidade da bupivacaína a $0,5 \%$ a $37^{\circ} \mathrm{C}$ é $0,9993 \mathrm{~g} \cdot \mathrm{ml}^{-110}$. A solução de bupivacaína a $0,15 \%$ foi selecionada por asse- gurar a hipobaricidade da solução ${ }^{11}$. Por definição, a baricidade é a relação entre a densidade da solução injetada e a densidade do LCR. A densidade média do LCR é de 1,00059 $\pm 0,00020 \mathrm{~g} \cdot \mathrm{ml}^{-112}$. A baricidade dos anestésicos locais pode ser diminuída pela diluição com água ${ }^{11}$. Adensidade da bupivacaína a $0,15 \%$ foi de $0,9954 \mathrm{~g} \cdot \mathrm{ml}^{-1}$ a $37^{\circ} \mathrm{C}$; portanto, hipobárica em todos os pacientes. Escolhemos a solução hipobárica pelo potencial perigo do acúmulo de solução hiperbárica na cauda eqüina ${ }^{13}$.

A utilização da raquianestesia unilateral tem aumentado bastante nos últimos anos, por produzir bloqueio motor de menor duração e em apenas um membro, proporcionando conforto do paciente, além de evitar as alterações hemodinâmicas. A bupivacaína a $0,5 \%$ isobárica é um anestésico local de longa duração de ação e, normalmente, emprega-se em sobredose para cirurgias ortopédicas de curta duração. A dose de $7,5 \mathrm{mg}$ de bupivacaína a $0,15 \%$ hipobárica associada ao fentanil proporcionou um tempo de recuperação de 2,94 horas $^{2}$, semelhante ao 2,96 horas obtido com $6,1 \mathrm{mg}$ de bupivacaína a $0,18 \%{ }^{9}$. Neste trabalho, ao reduzirmos a dose para $5 \mathrm{mg}$ de bupivacaína a $0,15 \%$ hipobárica, o tempo de recuperação foi de 2,32 horas, portanto $20 \%$ menor.

Diversos autores preferem a agulha ponta de lápis por direcionar o fluxo do anestésico com objetivo de produzir raquianestesia unilateral ${ }^{1,5,9,14-16}$. Entretanto, outros ${ }^{2,11,17,18}$ preferem a agulha ponta cortante (Quincke) pelo potencial perigo da injeção lenta em sentido caudal proporcionar altas concentrações de anestésicos hiperbáricos.

A agulha tipo Quincke proporciona um rápido aparecimento do LCR ${ }^{19}$, não causa má distribuição do anestésico local ${ }^{13} \mathrm{e}$ cursa com baixa incidência de cefaléia ${ }^{20}$. O tempo médio para atingir o nível máximo de analgesia com $5 \mathrm{ml}$ de bupivacaína a $0,5 \%$ foi de 17,3 minutos $^{21}$. O nível da analgesia da bupivacaína a $0,5 \%$ isobárica aumenta acima de 4 segmentos quando o paciente é colocado em cefaloaclive de $30^{\circ}$ por $80-115$ minutos $^{22}$. Neste estudo, os pacientes permaneceram na posição lateral por 20 minutos e com a mesa cirúrgica horizontal. O nível sensitivo no membro operado variou de $\mathrm{T}_{7}$ $a L_{1}$ e alguns pacientes apresentaram um certo grau de bloqueio no membro contralateral. Em trabalho com 7,5 mg associado ao fentanil e permanecendo pelos mesmos 20 minutos em decúbito lateral o nível variou de $T_{8}$ a $T_{12}$.

A raquianestesia unilateral pode ser realizada com soluções hipobáricas, isobáricas e hiperbáricas e a incidência de raquianestesia unilateral varia de trabalho para trabalho. Com soluções hipobáricas, a incidência de raquianestesia unilateral varia de $43 \%{ }^{9}$ a $71 \%{ }^{2}$. Com soluções isobáricas, sua incidência é de $37 \%{ }^{5}$. Com soluções hiperbáricas, depende da velocidade de injeção, sendo $33 \%{ }^{15} \mathrm{com}$ injeção rápida e $44 \%$ com injeção lenta ${ }^{15}$, havendo relatos de incidência de $83 \%{ }^{5}$. No presente trabalho, a incidência de raquianestesia unilateral foi de $75 \%$.

Um dos objetivos da raquianestesia unilateral é a diminuição da hipotensão arterial que pode ocorrer com a raquianestesia, fato confirmado quando se comparou a raquianestesia convencional com a raquianestesia assimétrica ${ }^{1}$. O uso de soluções hiperbáricas para obtenção de raquianestesia uni- 
lateral proporciona uma incidência de $10 \%{ }^{17}$ a $20 \%{ }^{15}$ de hipotensão arterial independentemente da velocidade de injeção. Já a utilização de soluções hipobáricas ${ }^{2,9,23,24}$ resulta numa menor incidência, o que foi confirmado neste trabalho. Da mesma forma, a incidência de hipotensão arterial foi significativamente maior após anestesia peridural quando se comparou com raquianestesia unilateral ${ }^{25}$.

Aregressão de dois segmentos com baixas doses de bupivacaína a $0,5 \%$ hiperbárica oscila entre $67^{1}$ e $99^{16}$ minutos. Já com o uso de bupivacaína hipobárica a regressão ocorreu em 53 minutos e a liberação do paciente entre 180 e 190 minutos ${ }^{26}$. No presente estudo, não foi avaliada a regressão de dois segmentos, mas sim o desaparecimento completo do bloqueio, que variou de 129 a 148 minutos. Desta forma, o uso de $5 \mathrm{mg}$ de bupivacaína hipobárica pode ser uma boa indicação para cirurgia ambulatorial.

Agulha ponta de lápis e injeção lenta são fatores que contribuem para o aparecimento de sintomas neurológicos transitórios, principalmente quando lidocaína hiperbárica é utiliza$\mathrm{da}^{27}$. Usando agulha ponta cortante e bupivacaína hipobárica nenhum caso de sintomas neurológicos transitórios foi observado neste estudo, que está de acordo com outros autores ${ }^{4,28}$ que avaliaram a influência da velocidade da injeção de bupivacaína hiperbárica a 0,5\% em distribuição lateral. A agulha ponta cortante cursa com uma baixa incidência de cefaléia ${ }^{20}$, fato confirmado neste estudo sem retirada de LCR, onde não ocorreu nenhum caso de cefaléia, da mesma forma em estudo anterior com retirada de 3 a $5 \mathrm{ml}^{2}$.

Alguns autores ${ }^{2,11,29}$ acreditam que um volume igual de LCR deva ser retirado para injeção de anestésico local hipobárico e obtenção de raquianestesia unilateral. Neste trabalho, foi demonstrado que não há necessidade de retirada de LCR, pois a incidência de $75 \%$ foi praticamente igual a obtida $(71 \%)^{2}$ quando se retirou um volume de 3 a $5 \mathrm{ml}$ de LCR. Concluindo, este estudo demonstrou que a raquianestesia unilateral com 5 mg de bupivacaína a $0,15 \%$ resulta num bloqueio motor intenso com regressão total de 139 minutos no lado operado e que não há necessidade de retirada de LCR. A principal vantagem desta forma de raquianestesia é a grande estabilidade cardiocirculatória, a satisfação do paciente em permanecer com um membro sem bloqueio e sua regressão rápida, podendo ser uma nova opção para cirurgia ambulatorial.

\section{AGRADECIMENTOS}

Agradecemos ao Prof. José Antônio Cordeiro pela valiosa orientação no estudo estatístico.

\section{Low Hypobaric Bupivacaine Doses for Unilateral Spinal Anesthesia}

Luiz Eduardo Imbelloni, TSA, M.D.; Lúcia Beato, TSA, M.D.; M.A. Gouveia, TSA, M.D.

\section{INTRODUCTION}

Spinal anesthesia is one of the oldest pain-relief methods for surgical patients. Surgical procedures involving just one lower limb are frequent, especially for outpatient procedures and orthopedic surgeries. In those situations, unilateral spinal anesthesia presents some advantages over conventional spinal anesthesia, like lower incidence of arterial hypotension ${ }^{1}$, faster recovery ${ }^{1}$ and increased patients' satisfaction ${ }^{2}$. There are several reasons to limit the upper sensory block level. Lower limb surgeries require low sensory block levels, resulting in higher cardiocirculatory stability ${ }^{3}$. Some undesirable effects, such as prolonged motor block, hemodynamic instability and urinary retention, may be prevented with low local anesthetic doses ${ }^{4,5}$. Lateral position, low local anesthetic doses, pencil point needle orientation and slow local anesthetic injection have been suggested to help unilateral spinal anesthesia achievement ${ }^{6,7}$. A previous study ${ }^{2}$ using $0.15 \%$ hypobaric bupivacaine, cutting point needle and 3 to $5 \mathrm{ml} \mathrm{CSF}$ removal has obtained unilateral spinal anesthesia in $71 \%$ of patients.

Our study aimed at evaluating the attempt to asymmetrically distribute anesthesia between the operated and contralateral limb, and its influence in the incidence of arterial hypotension during spinal anesthesia for lower limb orthopedic surgeries with low $0.15 \%$ hypobaric bupivacaine doses without CSF removal.

\section{METHODS}

After the Publication and Disclosure Board of Directors' approval and informed consent, 20 patients physical status ASA I and II, aged 21 to 79 years, undergoing elective and urgency orthopedic surgeries involving just one limb were studied. Exclusion criteria were hypovolemia, coagulation disorders, infections and refusal to the method. Monitoring consisted of non-invasive blood pressure, heart rate and pulse oximetry. No patient was premedicated. Venoclysis was performed in the operating room with lactated Ringer's solution, followed by 25 to $50 \mu \mathrm{g}$ fentanyl injection.

Patients were placed in the lateral position with the limb to be operated upwards. Spine was horizontally positioned before spinal puncture and the table was horizontally leveled (zero) prior to the procedure. Spinal puncture was paramedially performed at $L_{3}-L_{4}$ interspace with a $27 G$ Quincke needle followed by $5 \mathrm{mg}$ of $0.15 \%$ hypobaric bupivacaine (prepared as from $7.5 \mathrm{mg}$ of $0.5 \%$ isobaric bupivacaine plus $3.5 \mathrm{ml}$ bidistilled water) injection (Table I) at the rate of $1 \mathrm{ml} .15 \mathrm{~s}^{-1}$ with the needle's point facing toward the upper limb. Patients 
were maintained in this position for 20 minutes and then were placed in the supine position.

Table I - Evaluation of the Solution Injected in the CSF *

\begin{tabular}{lcc}
\hline & $20^{\circ} \mathrm{C}$ & $37^{\circ} \mathrm{C}$ \\
\hline Density $\left(\mathrm{g} \cdot \mathrm{ml}^{-1}\right)$ & 1.001 & 0.996 \\
Osmolarity $\left(\mathrm{mOsm} \cdot \mathrm{kg}^{-1}\right)$ & 149 & 149 \\
$\mathrm{pH}$ & 5.28 & 5.10 \\
Bupivacaine content $\left(\mathrm{mg} \cdot \mathrm{ml}^{-1}\right)$ & 7.585 & 7.585 \\
Water for injectables, as needed $(\mathrm{ml})$ & - & 3.5 \\
\hline
\end{tabular}

* Cristália Produtos Químicos e Farmacêuticos Ltda

Sensory block was evaluated by the loss of cold sensation (ice) and 27G pinprick, while motor block was evaluated by Bromage's modified scale $(0=$ no motor block and $3=$ total motor block). Sensory and motor blocks were evaluated in both limbs at 20, 40 and 60 minutes, as well as at surgery completion. Hemodynamic parameters were evaluated at 3-minute intervals during the first 15 minutes after spinal puncture, and then at 5-minute intervals until surgery completion.

Arterial hypotension was defined as $30 \%$ decrease of baseline values, and bradycardia as HR below $50 \mathrm{bpm}$. Arterial hypotension would be treated with $1 \mathrm{mg}$ intravenous ethylenephenilephrine and bradycardia with $0.5 \mathrm{mg}$ intravenous atropine. A pneumatic tourniquet was inflated during the procedure to $100 \mathrm{mmHg}$ above systolic blood pressure, and 2 L. min $^{-1}$ oxygen was administered through nasal catheter or Hudson's mask. Midazolam ( 1.5 to $3 \mathrm{mg}$ ) was administered after the second evaluation (40 $\mathrm{min}$ ).

Patients were followed up for 3 postoperative days to collect information on their satisfaction with the technique and to check for post-dural puncture headache or transient neurological symptoms (TNS), and for 30 days to check for permanent neurological complications.

Quantitative variables were analyzed by descriptive statistics and compared by Kruskal-Wallis non-parametric test. Blockade of both limbs was compared by non-parametric signal test. Significance level was established to $5 \%$.

\section{RESULTS}

Demographics data are shown in table II.

Table II - Demographics Data, Surgery and Sensory Block Duration

\begin{tabular}{lc}
\hline Age (years) * & $41.6 \pm 17$ \\
Weight $(\mathrm{kg}){ }^{*}$ & $65.6 \pm 16.1$ \\
Height $(\mathrm{cm}){ }^{*}$ & $163.3 \pm 8.2$ \\
Gender & \\
$\quad$ Female & 12 \\
$\quad$ Male & 8 \\
Surgery duration (hours) * & $1.2 \pm 0.2$ \\
Blockade duration (hours) * & $2.32 \pm 0.16$ \\
\hline
\end{tabular}

* Values expressed in Mean \pm SD

Revista Brasileira de Anestesiologia

Vol. 53, N 5, Setembro - Outubro, 2003
Total unilateral block was seen in $75 \%$ of patients. Total mean recovery time was 2.32 hours, suffering no influence of gen$\operatorname{der}(p=0.21)$ or surgery duration $(p=0.88)$.

Sensory block spread in the operated limb side varied from $T_{7}$ to $L_{1}$ with mode equal to $T_{12}$. At 20 minutes, 4 patients presented contralateral limb sensory block varying from $T_{10}$ to $L_{1}$. At 40 minutes, one more patient has referred sensory block at $\mathrm{T}_{12}$. So, there has been unilateral sensory block in 15 patients $(75 \%)$, and bilateral block in 5 patients (25\%). Operated limb blockade was significantly higher.

All patients showed total motor block in the operated limb. One patient had total motor block in the contralateral limb. There has been motor block level 2 in two patients and level 1 in two patients (Figure 1). There has been unilateral motor block in 15 patients (75\%). Operated limb motor block was higher as compared to contralateral limb in all evaluations.

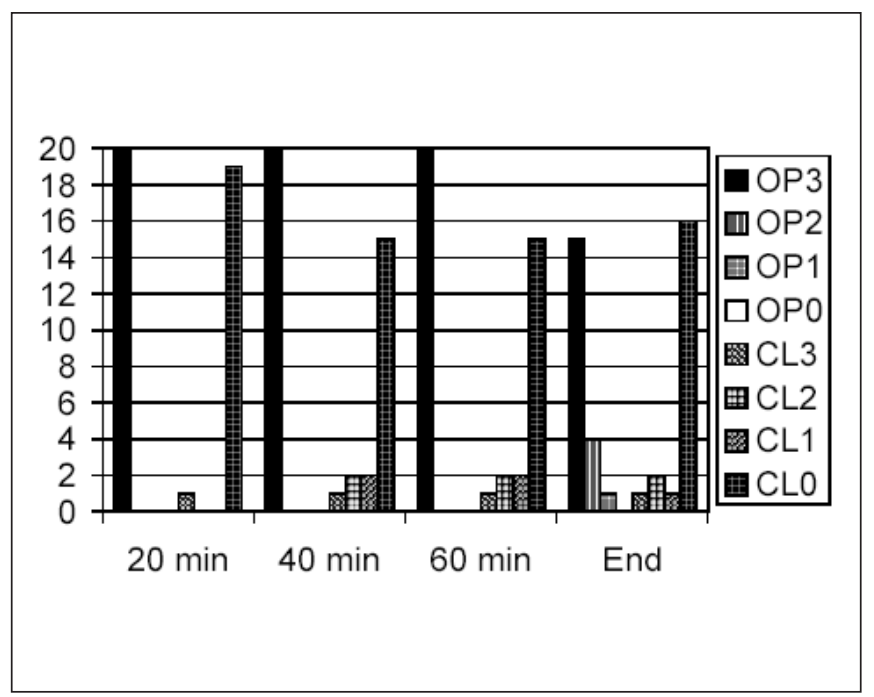

Figure 1 - Motor Block on the Operated and Contralateral Sides in Different Times

* $0.15 \%$ bupivacaine kindly produced and evaluated by Cristália Produtos Farmacêuticos Ltda

Spinal anesthesia was satisfactory for all patients during the procedure and no patient required general anesthesia association. One patient has referred tourniquet pain at 85 minutes of surgery. There were no blood pressure and heart rate changes in any patient. There has been no urinary retention and no patient developed post-dural puncture headache. There has been no reported back, buttocks or legs pain which could be considered transient neurological symptoms, nor late neurological complications.

There was no anesthetic failure. All patients were happy with the technique. All 15 patients with unilateral anesthesia reported a comfortable sensation of feeling the contralateral limb.

\section{DISCUSSION}

Low hypobaric bupivacaine doses $(5 \mathrm{mg})$ at the injection rate of $1 \mathrm{ml} .15 \mathrm{~s}^{-1}$ through a $27 \mathrm{G}$ Quincke needle have induced to- 
tal unilateral block in $75 \%$ of patients. Unilateral spinal anesthesia has shown to be safe and effective in single-limb orthopedic surgeries, decreasing hemodynamic effects of neuraxial blocks ${ }^{1,2,8}$ and increasing patients' satisfaction with the technique.

Density of $0.5 \%$ bupivacaine at $37^{\circ} \mathrm{C}$ is $0.9993 \mathrm{~g} \cdot \mathrm{ml}^{-1}{ }^{10}$. Bupivacaine solution at $0.15 \%$ was selected for assuring solution's hypobaricity ${ }^{11}$. By definition, baricity is the relationship between injected solution density and CSF density. Mean CSF density is $1.00059 \pm 0.00020 \mathrm{~g} \cdot \mathrm{ml}^{-112}$. Local anesthetics baricity may be decreased by water dilution ${ }^{11}$. Bupivacaine at $0.15 \%$ baricity was $0.9954 \mathrm{~g} \cdot \mathrm{ml}^{-1}$ at $37^{\circ} \mathrm{C}$, thus hypobaric for all patients. Hypobaric solution was chosen due to the risk of hyperbaric solution accumulation in the cauda equina ${ }^{13}$

Unilateral spinal anesthesia is becoming increasingly popular in recent years for producing shorter duration motor block in just one limb, allowing comfort to patients in addition to preventing hemodynamic changes. Isobaric $0.5 \%$ bupivacaine is a long-lasting local anesthetic and, being frequently overdosed for short orthopedic surgeries. The dose of $7.5 \mathrm{mg}$ of $0.15 \%$ hypobaric bupivacaine associated to fentanyl has provided a recovery time of 2.94 hours $^{2}$, similar to 2.96 hours obtained with $6.1 \mathrm{mg}$ of $0.18 \%$ bupivacaine ${ }^{9}$. Our study has decreased the dose to $5 \mathrm{mg}$ of $0.15 \%$ hypobaric bupivacaine reflecting a recovery time of 2.32 hours, or $20 \%$ shorter. Several authors prefer pencil point needles to orient anesthetic flow and thus help promoting unilateral spinal anesthesia $1,5,9,14-16$. Others ${ }^{2,11,17,18}$, however, prefer cutting point needles (Quincke) for the potential danger of slow caudal injection producing high hyperbaric anesthetic concentrations. Quincke needles allow a fast CSF return ${ }^{19}$, do not lead to local anesthetic mal-distribution ${ }^{13}$ and are associated to low headache incidence ${ }^{20}$. In one study, mean time to reach maximum analgesia with $5 \mathrm{ml}$ of $0.5 \%$ bupivacaine was 17.3 minutes ${ }^{21}$. Another one showed that analgesic level of $0.5 \%$ isobaric bupivacaine increases above 4 segments when patients are placed at $30^{\circ}$ head-up for $80-115$ minutes ${ }^{22}$. In our study, patients remained in the lateral position for 20 minutes with the surgical table in the horizontal position. Sensory level in the operated limb varied from $T_{7}$ to $L_{1}$, and some patients presented a certain degree of contralateral limb blockade. In a study with $7.5 \mathrm{mg}$ associated to fentanyl and remaining for the same 20 minutes in the lateral position, the level has varied from $T_{8}$ to $T_{12}$.

Unilateral anesthesia may be achieved with hypobaric, isobaric or hyperbaric solutions, and the incidence of unilateral anesthesia varies in a study-to-study basis. The incidence of unilateral anesthesia with hypobaric solutions varies from $43 \%{ }^{9}$ to $71 \%{ }^{2}$. Its incidence is $37 \%{ }^{5}$ with isobaric solutions, and with hyperbaric solutions it depends on injection speed, being $33 \%{ }^{15}$ with fast injections and $44 \%{ }^{15}$ with slow injections, with studies reporting up to $83 \%{ }^{5}$. The incidence of unilateral anesthesia in our study was $75 \%$.

One of the objectives of unilateral anesthesia is to decrease arterial hypotension, which may be present during spinal anesthesia, and this was confirmed when conven- tional spinal anesthesia was compared to asymmetric spinal anesthesia ${ }^{1}$. Hyperbaric unilateral spinal anesthesia induces $10 \%{ }^{17}$ to $20 \%{ }^{15}$ arterial hypotension, regardless of injection speed. Hypobaric solutions ${ }^{2,9,23,24}$ result in a lower incidence, what was confirmed in our study. Similarly, the incidence of arterial hypotension was significantly higher after epidural anesthesia as compared to unilateral spinal anesthesia ${ }^{25}$.

Two segments regression time with low doses of $0.5 \%$ hyperbaric bupivacaine varies between $67^{1}$ and $99^{16}$ minutes. With hypobaric bupivacaine, regression has occurred in 53 minutes and patients were discharged after 180 to 190 minutes ${ }^{26}$. Our study has not evaluated two-segment regression, but rather total blockade recovery, which has varied from 129 to 148 minutes. So, 5 mg hypobaric bupivacaine may be a good indication for outpatient procedures.

Pencil point needles and slow injections are factors related to transient neurological symptoms, especially with hyperbaric lidocaine ${ }^{27}$. No transient neurological symptoms were observed in this study with cutting point needles and hypobaric bupivacaine, which is in line with other authors ${ }^{4,28}$ who have evaluated the influence of $0.5 \%$ hyperbaric bupivacaine injection speed in lateral distribution.

Cutting point needles course with low headache incidence ${ }^{20}$, what was confirmed by our study with no CSF removal (no headache observed) and similar to a previous study with 3 to $5 \mathrm{ml} \mathrm{CSF}$ removal ${ }^{2}$.

Some authors ${ }^{2,11,29}$ believe that an equal CSF volume should be removed prior to the hypobaric local anesthetic injection for unilateral anesthesia. Our study has shown that there is no need for that, since our $75 \%$ success rate was virtually the same as when 3 to $5 \mathrm{ml} \mathrm{CSF}$ were removed $(71 \%)^{2}$.

In conclusion, our study has shown that unilateral spinal anesthesia with $5 \mathrm{mg}$ of $0.15 \%$ bupivacaine results in an intense motor block with total regression time of 139 minutes on the operated side, and that there is no need for prior CSF removal. The great advantages of this type of spinal anesthesia are the high cardiocirculatory stability, patients' satisfaction in remaining with an unblocked limb and fast recovery, what renders it a good option for outpatient procedures.

\section{ACKNOWLEDGEMENT}

We acknowledge Prof. José Antonio Cordeiro for his invaluable orientation during our statistical studies.

\section{REFERÊNCIAS - REFERENCES}

01. Casati A, Fanelli G, Aldegheri G et al - Frequency of hypotension during conventional or asymmetric hyperbaric spinal block. Reg Anesth Pain Med, 1999;24:214-219.

02. Imbelloni LE, Beato L, Gouveia MA - Raquianestesia unilateral com bupivacaína hipobárica. Rev Bras Anestesiol, 2002;52: 542-548 
03. Imbelloni LE - O Uso Racional da Raquianestesia, em: Imbelloni LE - Tratado de Anestesia Raquidiana, Medidática Informática Ltda, Curitiba, 2001;8:74.

04. Pittoni G, Toffoletto F, Calcarella G et al - Spinal anesthesia in outpatient knee surgery: 22-gauge versus 25-gauge Sprotte needle. Anesth Analg, 1995;81:73-79.

05. Kuusniemi KS, Pihlajamäki KK, Pitkänen MT - A low dose of plain or hyperbaric bupivacaine for unilateral spinal anesthesia. Reg Anesth Pain Med, 2000;25:605-610.

06. Tanasichuk MA, Schultz EA, Matthews JH et al - Spinal hemianalgesia: An evaluation of a method, its applicability, and influence of the incidence of hypotension. Anesthesiology, 1961;22:74-85

07. Casati A, Fanelli G, Cappelleri G et al - Low dose hyperbaric bupivacaine for unilateral spinal anaesthesia. Can J Anaesth, 1998;45:850-854

08. Casati A, Fanelli G, Berti M et al - Cardiac performance during unilateral lumbar spinal block after crystalloid preload. Can J Anaesth, 1997;44:623-628.

09. Kuusniemi KS, Pihlajamäki KK, Pitkänen MT et al - Low-dose bupivacaine: a comparison of hypobaric and near isobaric solutions for arthroscopic surgery of the knee. Anaesthesia, 1999; 54:540-545.

10. Cangiani LM - Determinação da densidade e da baricidade das misturas para anestesia subaracnóidea. Rev Bras Anestesiol, 2000;50:92-94

11. Gouveia MA, Labrunie GM - Raquianestesia hipobárica com bupivacaína 0,15\%. Rev Bras Anestesiol, 1985;35:519-521.

12. Lui ACP, Polis TZ, Cicutti NJ - Densities of cerebrospinal fluid and spinal anaesthetic solutions in surgical patients at body temperature. Can J Anesth, 1998;45:297-303.

13. Holman St J, Robinson RA, Beardsley D et al - Hyperbaric dye solution distribution characteristics after pencil-point needle injection in a spinal cord model. Anesthesiology, 1997;86: 966-973.

14. Enk D, Prien T, Van Aken $\mathrm{H}$ et al - Success rate of unilateral spinal anesthesia is dependent on injection flow. Reg Anesth Pain Med, 2001;26:420-427.

15. Casati A, Fanelli G, Cappelleri G et al - Effects of speed of intrathecal injection on unilateral spinal block by $1 \%$ hyperbaric bupivacaine. A randomized, double-blind study. Minerva Anestesiol, 1999;65:5-10.

16. Fanelli G, Borghi B, Casati A et al - Unilateral bupivacaine spinal anesthesia for outpatient knee arthroscopy. Can J Anaesth, 2000;47:746-751.

17. Esmaoglu A, Boyaci A, Ersoy Ö et al - Unilateral spinal anaesthesia with hyperbaric bupivacaine. Acta Anaesthesiol Scand, 1998;42:1083-1087.

18. Iselin-Chaves I, Van Gessel EF, Donald F et al - The effects of solution concentration and epinephrine on lateral distribution of hyperbaric tetracaine spinal anesthesia. Anesth Analg, 1996;83:755-759.

19. Imbelloni LE, Carneiro ANG, Sobral MGC - Tempo de gotejamento de líquido cefalorraquidiano com agulhas espinhais de fino calibre. Rev Bras Anestesiol, 1995;45: 155-158.

20. Imbelloni LE, Sobral MGC, Carneiro ANG - Cefaléia pós-raquianestesia e o desenho das agulhas. Experiência com 5050 casos. Rev Bras Anestesiol, 2001;51:43-52.
21. Cameron AE, Arnold RW, Ghoris MW et al - Spinal analgesia us ing bupivacaína 0.5\% plain. Anaesthesia, 1981;36:318-322.

22. Niemi L, Tuominen M, Pitkänen $M$ et al - Effect of late posture change on the level of spinal anaesthesia with plain bupivacaine. Br J Anaesth, 1993;71:807-809.

23. Kuusniemi KS, Pihlajamäki KK, Pitkänen MT et al - A low-dose hypobaric bupivacaine spinal for knee arthroscopies. Reg Anesth, 1997:22:534-538.

24. Kuusniemi KS, Pihlajamäki KK, Kirvelä OA et al - Spinal anesthesia with hypobaric bupivacaine for knee arthroscopies: Effect of posture on motor block. Reg Anesth Pain Med, 2001;26:30-34.

25. Kuusniemi KS, Pihlajamäki KK, Irjala JK et al - Restricted spinal anesthesia for ambulatory surgery: a pilot study. Eur J Anesth, 1999;16:2-6.

26. Ben-David B, Levin H, Solomon E et al - Spinal bupivacaine in ambulatory surgery: the effect of saline dilution. Anesth Analg, 1996;83:716-720.

27. Beardsley D, Holman S, Gant R et al - Transient neurologic deficit after spinal anesthesia: Local anesthetic maldistribution with pencil-point needles? Anesth Analg, 1995;81:314-320.

28. Casati A, Fanelli G, Cappelleri G et al - Does speed of intrathecal injection affect the distribution of $0.5 \%$ hyperbaric bupivacaine? Br J Anesth, 1998;81:355-357.

29. Gouveia MA, Labrunie GM - Raquianestesia hipobárica com tetracaína 0,1\%. Rev Bras Anestesiol, 1985;35:232-233.

\section{RESUMEN}

Imbelloni LE, Beato L, Gouveia MA - Dosis Bajas de Bupivacaína Hipobárica para Raquianestesia Unilateral

JUSTIFICATIVA Y OBJETIVOS: Para evitar alteraciones hemodinámicas, obtener recuperación más rápida y limitar la dispersión cefálica de la raquianestesia apenas en el miembro operado fue realizado este estudio con bupivacaína a 0,15\%, con objetivo de obtenerse raquianestesia unilateral.

MÉTODO: Raquianestesia con $3,3 \mathrm{ml}$ de bupivacaína hipobárica a 0,15\% (5 mg) fue realizada a través de aguja $27 \mathrm{G}$ Quincke en 20 pacientes estado físico ASA I y II sometidos a cirugías ortopédicas. La punción subaracnóidea fue realizada por via lateral con el paciente en decúbito lateral, con el miembro a ser operado vuelto para arriba, y 3,3 $\mathrm{ml}$ de bupivacaína hipobárica fueron inyectados en la velocidad de 1 $\mathrm{ml}$ a cada 15 segundos. Bloqueo sensitivo y motor (picada de aguja y escala de 0 a 3) fueron comparados entre los lados que serian operados y el contralateral.

RESULTADOS: Los bloqueos motor y sensitivo entre el lado operado y el contralateral fueron significativamente diferentes en todos los tiempos evaluados. Raquianestesia unilateral fue obtenida en $75 \%$ de los pacientes. Estabilidad hemodinámica fue observada en todos los pacientes. Ningún paciente desarrolló cefalea pos-raquianestesia.

CONCLUSIONES: La bupivacaína hipobárica a 0,15\% en dosis de $5 \mathrm{mg}$ proporciona un predominante bloqueo unilateral. Veinte minutos son suficientes para su instalación. La principal ventaja de la raquianestesia unilateral es la estabilidad hemodinámica. 